\title{
A modified frozen elephant trunk hybrid device to facilitate supra-aortic trunk anastomosis
}

\author{
Paolo Masiello ${ }^{1}$, Generoso Mastrogiovanni ${ }^{1}$, Pierpaolo Chivasso ${ }^{2}$, Donato Triggiani $^{1}$, \\ Francesco Cafarelli ${ }^{1}$, and Severino Iesu ${ }^{1}$ \\ ${ }^{1}$ University Hospital 'San Giovanni di Dio e Ruggi d'Aragona' \\ ${ }^{2}$ Azienda Ospedaliera Universitaria 'San Giovanni di Dio e Ruggi d'Aragona'
}

September 11, 2020

\begin{abstract}
Reimplantation of the supra-aortic vessels can be challenging with Thoraflex Hybrid. A device modification made the vessel lengths more appropriate and the position of the neo-vessels in the chest avoided malpositioning and kinking and facilitated sternum closure; this may improve operating times as well as allowing complete and continuous cerebral trivascular perfusion and corrects positioning of the intrathoracic vessels.
\end{abstract}

\section{Key words}

Frozen elephant trunk, customization, aortic arch, hybrid surgery

\section{Main text}

\section{Introduction}

Both acute and chronic aortic pathologies are often associated with descending aorta disease. The Frozen Elephant Trunk (FET) hybrid procedure has developed to treat both segments, sometimes with a definitive aortic reconstruction in one stage or in anticipation of subsequent completion with thoracic endovascular repair (TEVAR). Thoraflex Hybrid (Terumo Aortic) is a dedicated FET device that joins a distal endovascular nitinol ringed stent-graft with a proximal 4-branched surgical graft and has been used in acute and chronic settings to reduce circulatory arrest time and improve cerebral and systemic perfusion. ${ }^{1}$ We have implanted over 70 Thoraflex Hybrid devices and been challenged by reimplantation of the supra-aortic trunk (SAT) vessels, expecially the left carotid (LCA) and left subclavian (LSA) arteries, their chest positioning, and management of cerebral perfusion. To address this, we requested a custom-made configuration and describe here the first experience.

\section{Case Report}

A 72-year-old male with adenocarcinoma but no other known major comorbidities was scheduled for right nephrectomy; a routine echocardiography showed an undiagnosed dilated ascending aorta with aortic insufficiency. Subsequent computed tomographic angiography (CTA) showed a dilated ascending aorta (58 $\mathrm{mm}$ ) and arch (particularly in zone 1, $49 \mathrm{~mm}$ ), as well as proximal thoracic aorta $(36 \mathrm{~mm})$. The aortic root was normal but a dilated sinotubular junction resulted in a moderate-to-severe aortic insufficiency. Biventricular contractility was preserved and there was no coronary artery pathology. The patient underwent uncomplicated nephrectomy and came back for aortic surgery 6 months later.

A 28/30 mm diameter Thoraflex Hybrid with a stented length of $100 \mathrm{~mm}$ was modified to have only two branches: one main $(16 \mathrm{~mm})$ for SAT reimplantation, and one lateral $(10 \mathrm{~mm})$ for systemic perfusion. A 
separate trifurcated Gelweave? surgical graft (10/8/8 - $16 \mathrm{~mm}$ ) (Terumo Aortic) with a 10-mm side perfusion branch was used to connect the SAT vessels (Figure 1).

Median sternotomy was performed and the usual incision was extended in a small bilateral supra-clavicular cervicotomy to improve SAT access and harvesting. Central cannulation using a 10-mm side graft was via the right intrathoracic subclavian artery (RSA). The right atrium and right superior pulmonary vein were cannulated for venous return and venting. A homemade 4-branched perfusion circuit was used for extracorporeal circulation (ECC) and cerebral antegrade perfusion (Figure 2). Four branches of equal diameter allow systemic perfusion by a single pump head while connected to RSA, LSA, LCA and prosthesis branch. Perfusion is kept at full flow for ECC and redistributed depending on physiological systemic resistence; then lowered to $10-12 \mathrm{~mL} / \mathrm{kg} / \mathrm{min}$ when the innominate is clamped for isolated cerebral perfusion.

The patient was cooled to $26^{\circ} \mathrm{C}$ for hypothermic circulatory arrest (HCA). On beating heart during the initial cooling phase, LCA and LSA were isolated, resected and anastomosed to the two lateral branches of the Gelweave prosthesis; cannulation and perfusion via the graft's side branch allowed complete antegrade cerebral perfusion.

After debranching and at $30^{\circ} \mathrm{C}$ core temperature, the aorta was cross-clamped and opened and Custodiol? cardioplegia administered into the coronary ostia. The aortic valve, unsuitable for repair, was replaced with a Inspiris Resilia 25-mm prosthesis (Carpentier Edwards). At 26degC, the innominate artery was clamped, systemic circulation stopped, and selective antegrade perfusion began at $12 \mathrm{~mL} / \mathrm{kg} / \mathrm{min}$. The arch was then opened and the landing zone (zone 2) was reinforced with a teflon strip. The distal stent-graft portion was deployed in the descending aorta and its reinforced collar was sutured to the aortic isthmus; after cannulation of the lateral branch systemic perfusion was resumed and rewarming started. The proximal anastomosis was then completed and cross-clamp released. The innominate was detached and anastomosed to the last branch of the second prosthesis. Finally, the main bodies of the FET and surgical grafts were anastomosed together.

At the end of the procedure, we found an extensive RSA wall lesion which we resected and anastomosed to the innominate with a 8-mm interposed graft: ECC, aortic clamping, and HCA times were 230, 118, and 27 minutes, respectively (Figure 3). The patient was extubated after 12 hours without any neurological damage. On postoperative day (POD) 4, he developed temporary respiratory insufficiency requiring tracheostomy. A moderate renal insufficiency developed and reverted after three days of continuous veno-venous hemofiltration. He was discharged from intensive care on POD 14 and transferred to rehabilitation without any other major problem.

\section{Discussion}

The frozen elephant trunk (FET) technique has rapidly gained acceptance and - despite its complexityrepresents the treatment of choice for chronic aortic disease in many centers, ${ }^{2,3}$ but its use in acute dissection is still debated. ${ }^{4-6}$ Since 2018, Thoraflex Hybrid is our FET device of choice because of the advantages of the branched plexus configuration but LSA anastomosis remains its Achilles heel. ${ }^{7}$ To address this, we debranch and selectively cannulate the LSA and LCA with interposition of $10 / 8 \mathrm{~mm}$ Dacron prosthesis avoiding direct cannulation and optimizing bilateral cerebral perfusion (always trivascular). However, it can be difficult to trim the graft length and position it in the chest often resulting in redundant or kinked branch vessels, particularly in small chests, and difficulty in closing the sternum.

In this case, the second surgical graft was free to move and we made a direct end-to-end anastomosis while perfusing the vessels via the side branch. Cerebral perfusion is complete and continuous in our standard practice; HCA and operation times are shortened and probably contribute to a low incidence of major stroke, even in acute dissections $(2.3 \%$ [1/42]; $1.3 \%,[1 / 74]$ all implants, data pending publication) compared to the literature $(5-14 \%) .^{7,8}$ With this device modification, the vessel lengths are more appropriate and the position of the neo-vessels in the chest avoids malpositioning and/or kinking, and facilitates sternum closure.

\section{Conclusion}


A modification to the standard Thoraflex Hybrid design allowed improvement in operating times, complete and continuous cerebral trivascular perfusion, and correct positioning of the intrathoracic vessels.

\section{Disclosure Statement}

No conflict of interest to declare

\section{Author contributions}

Concept/design PM,GM

Drafting article: PM,PC

Critical revision of article: SI,GM,FC,DT

Ethics approval and consent to participate : ethical approval was obtained from the director of A.O.U. San Giovanni di Dio e Ruggi D'Aragona Hospital. For case reports is not necessary a formal approval in this hospital

Consent to publish : the written consent for publication was released by the patient

\section{References}

1. Beckmann E, Martens A, Korte W, Kaufeld T, Krueger H, Haverich A, et al. Open total arch replacement with trifurcated graft and frozen elephant trunk. Ann Cardiothorac Surg 2020;9(3):170-177

2. Tsagakis K, Wendt D, Dimitriou AM, Thielmann M, Shehada S-E, El Gabry M, et al. The frozen elephant trunk treatment is the operation of choice for all kinds of arch disease. J Cardiovasc Surg (Torino). 2018;59(4):540-6

3. Shrestha M, Bachet J, Bavaria J, Carrel TP, De Paulis R, Di Bartolomeo R, et al. Current status and recommendations for use of the frozen elephant trunk technique: a position paper by the Vascular Domain of EACTS. Eur J Cardiothorac Surg 47 (2015) 759-769

4. Shrestha M, Martens A, Kruger H, Maeding I, Ius F, Fleissner F, et al. Total aortic arch replacement with the elephant trunk technique: single-centre 30-year results. Eur J Cardiothorac Surg. 2014 Feb;45(2):289-95; discussion 295-296

5. Ouzounian M, David TE. Total aortic repair for acute type A dissection: Not every patient; not every surgeon. Journal Thorac Cardiovasc Surg 2018 Sep 21:1-2

6. Morshuis WJ. Why to be cautious with the use of the frozen elephant trunk in acute type A aortic dissection. J Vis Surg 2018; 4:73

7. Chu MWA, Losenno KL, Dubois LA, Jones PM, Ouzounian M, Whitlock R, et al. Early Clinical Outcomes of Hybrid Arch Frozen Elephant Trunk Repair With the Thoraflex Hybrid Graft. Ann Thorac Surg 2019;107:47-53

8. Shrestha M, Kaufeld T, Beckmann E, Fleissner F, Umminger J, Alhadi FA, et al. Total aortic arch replacement with a novel 4-branched frozen elephant trunk prosthesis: Single-center results of the first 100 patients. J Thorac Cardiovasc Surg 2016;152:148-59

\section{Figure legends}

Figure 1. A custom-made Thoraflex Hybrid: A, cerebral vessels branch (16 mm); B, perfusion side branch $(10 \mathrm{~mm})$ and a Gelweave surgical graft:1, perfusion side branch $(10 \mathrm{~mm}) ; 2$, innominate branch $(10 \mathrm{~mm}) ; 3$, left carotid branch $(8 \mathrm{~mm}) ; 4$, left subclavian branch $(8 \mathrm{~mm})$

Figure 2 . Homemade perfusion system driven by a single pump: 1, right intrathoracic subclavian artery perfusion (systemic perfusion); 2, left subclavian artery perfusion; 3, left carotid artery perfusion; 4, side branch systemic perfusion

Figure 3 . Final result showing combined surgical and hybrid implanted grafts 

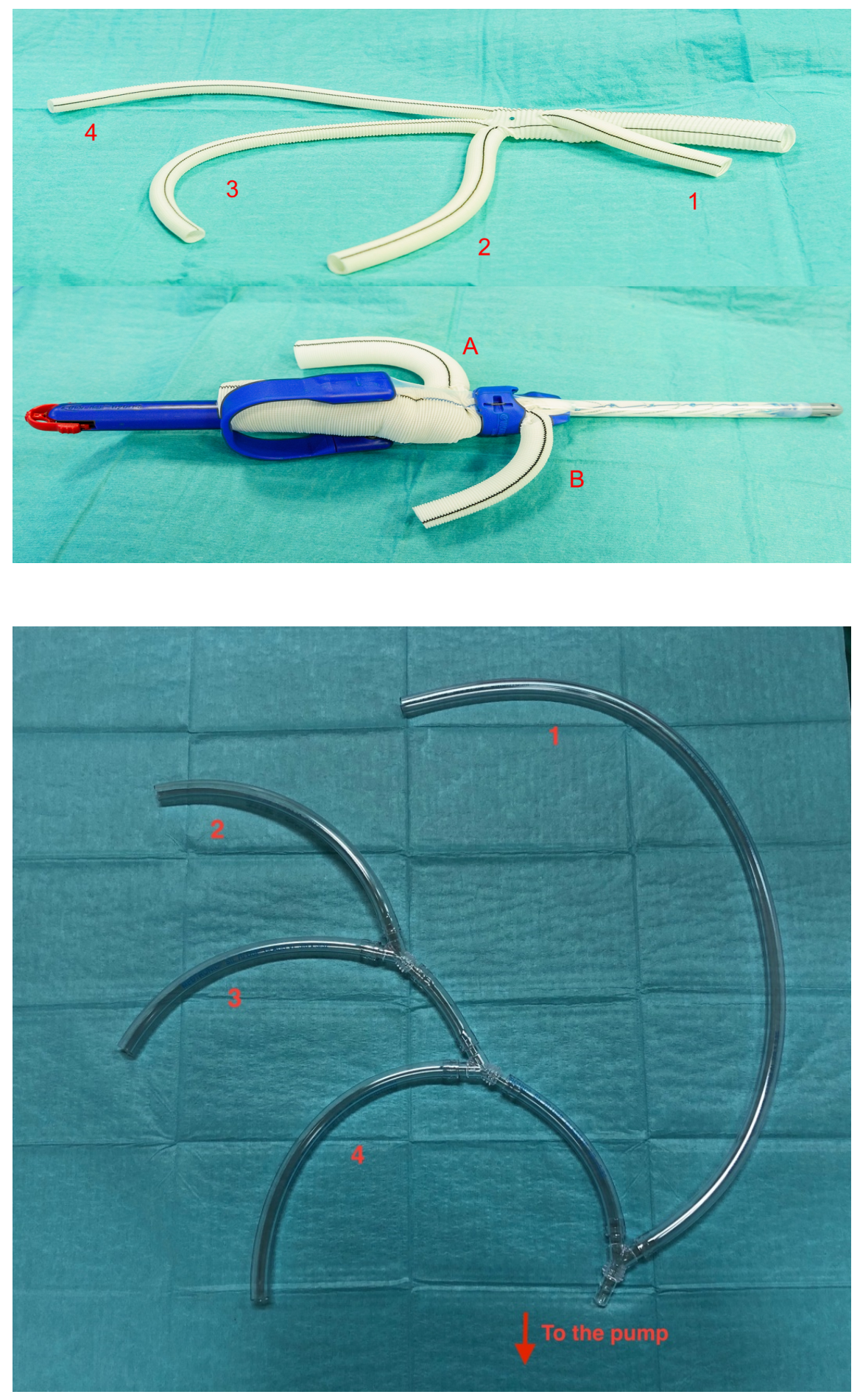


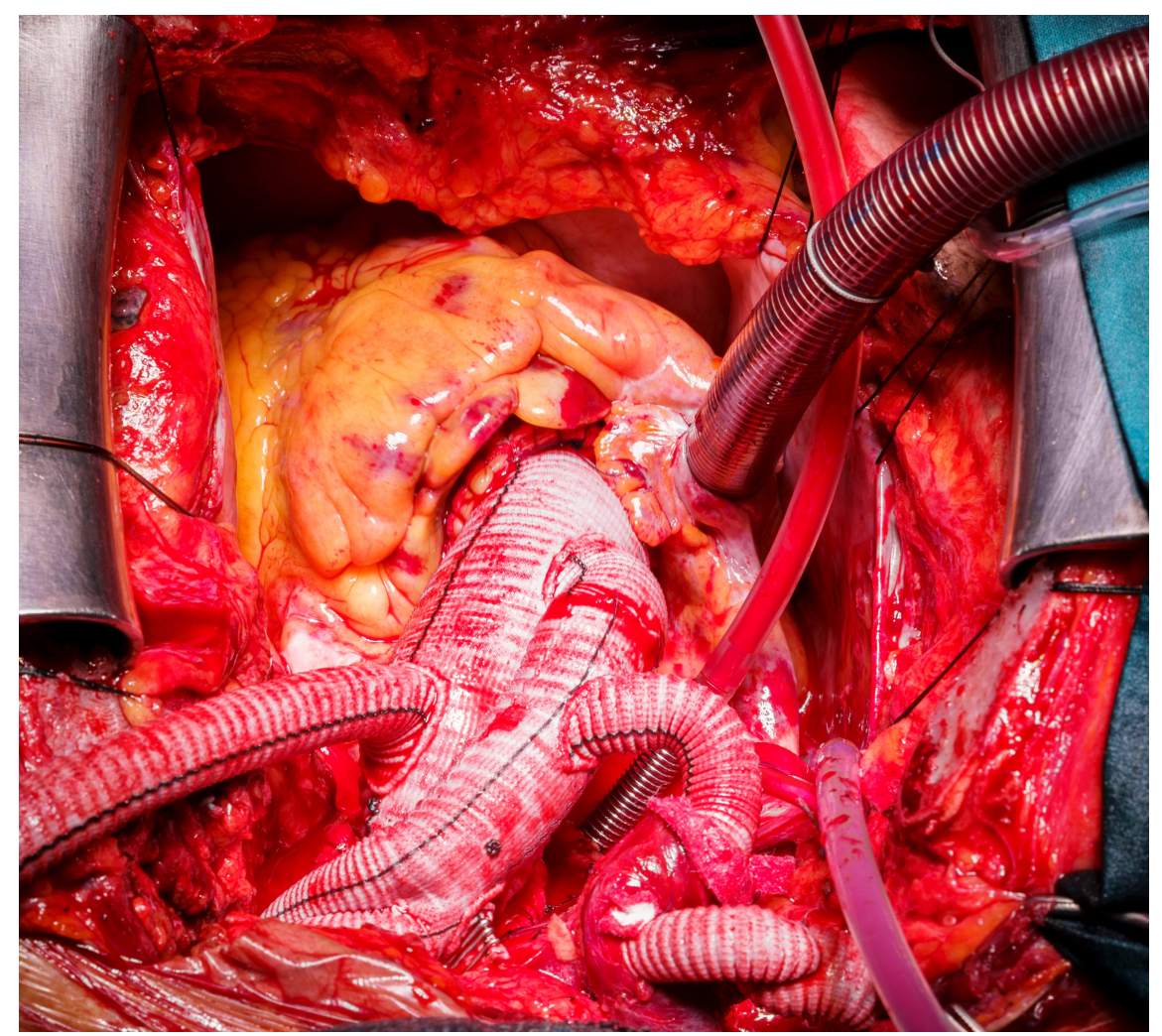

